Le Lait, 1986, 66 (3), 247-256

\title{
Influence de la phosphorylation sur le comportement des peptides trypsiques de caséine $\beta$ bovine en chromatographie liquide haute performance en phase inverse
}

\author{
C. CARLES et B. RIBADEAU DUMAS \\ Institut National de la Recherche Agronomique \\ C.N.R.Z., 78350 Jouy-en-Josas, France
}

\begin{abstract}
Résumé
La déphosphorylation complète de la caséine $\beta$ bovine, étudiée par électrophorèse et dosage du phosphore, a été réalisée par la phosphatase acide de pomme de terre. Cet enzyme ne réagissait pas avec le substrat quand ce dernier était précipité. Il a été montré par ailleurs que la préparation commerciale utilisée était contaminée par une protéase à sérine.

Les hydrolysats tryptiques obtenus à partir de la protéine native ou totalement déphosphorylée ont été fractionnés et comparés en chromatographie liquide haute performance en phase inverse (C18). Seuls les phosphopeptides et les peptides déphosphorylés homologues avaient des temps de rétention différents, mais les variations observées, de 0,4 à 1,9 min par groupement phosphate, dépendaient du peptide considéré. Ainsi, la théorie selon laquelle, dans des conditions d'élution bien définies, la rétention d'un peptide peut être considérée comme la somme arithmétique des contributions de chaque résidu, des groupements terminaux et des substitutions latérales, ne peut pas être appliquée dans ce cas précis.
\end{abstract}

Mots clés : Déphosphorylation - Caséine $\beta$ - Phase inverse - Phosphopeptides - HPLC.

\section{Summary}

Influence of phosphorylation on the behaviour of bovine $\beta$-casein tryptic peptides obtained by reverse phase high performance liquid chromatography

Using electrophoretic technics and phosphorus determinations, the total dephosphorylation of bovine $\beta$-casein has been performed by the action of potatoe acid phosphatase. The enzyme did not react with the substrate when it became insoluble. Moreover it has been observed that the commercial preparation used was contaminated with a serine proteinase. 
Tryptic hydrolysates of the native or totally dephosphorylated protein have been studied by reverse phase high performance liquid chromatography. Peptide mappings were similar except for phosphopeptides and their dephosphocounterparts. For these particular peptides, observed differences in retention time were between 0.4 and $0.9 \mathrm{~min}$ per phosphate group. Therefore the theory which predicts the retention time of a peptide from the sum of the contributions of each residue, terminal groups and lateral substitutions does not apply here.

Key words: Dephosphorylation - $\beta$-casein - Reverse phase - Phosphopeptides - HPLC.

$\begin{array}{ll}\text { TPCK } & : \text { Tosyl Phenylalanine Chloromethyl Ketone. } \\ \text { TCA } & : \text { Acide Trichloracétique. } \\ \text { RP-HPLC } & : \text { Reverse Phase High Performance Liquid Chromatography. } \\ \text { E/S } & : \text { Enzyme/substrat }(P / P) . \\ \text { EDTA } & : \text { Acide Ethylène Diaminetétraacétique. } \\ P M S F & : \text { Phényl Méthane Sulfonyl Fluorure. } \\ t_{r} & : \text { Temps de rétention. }\end{array}$

\section{Introduction}

On a recours de plus en plus fréquemment à l'ADNc pour déterminer indirectement la structure primaire de la protéine correspondante. Si cette approche est fructueuse, elle ne peut cependant pas être utilisée pour mettre en évidence les modifications post-traductionnelles. En ce domaine, l'étude de la protéine ellemême reste nécessaire. Nous nous sommes intéressés à un type particulier de modification : la phosphorylation. Le but principal de cette étude était de voir si la comparaison d'hydrolysats de caséine $\beta$ bovine phosphorylée et déphosphorylée permettait de localiser les résidus substitués par des groupements phosphate et d'en apprécier le nombre.

Par ailleurs, le rôle de plus en plus important des hydrolysats de protéines laitières utilisées à des fins nutritionnelles augmente encore l'intérêt de méthodes permettant la caractérisation de ce type d'échantillon.

Cette étude nécessitait auparavant la mise au point de conditions de déphosphorylation totale de la protéine. Nous avons employé à cet effet la phosphatase acide de pomme de terre (EC. 3.1.3.2.), en changeant les conditions de réaction initialement décrites par ALVAREz (1962) et utilisées par BRIGNON et al. (1977) lors de leurs études sur la déphosphorylation de la caséine $\alpha_{\mathbb{S}_{2}}$.

Des hydrolysats trypsiques de caséine $\beta$ native et totalement déphosphorylée ont été ensuite comparés par RP-HPLC.

\section{Matériel et méthodes}

Préparation de la caséine $\beta$

La caséine $\beta \mathrm{A}^{1}$ était préparée selon Mercier et al. (1968) à partir du lait d'une vache homozygote aux 4 loci des caséines. La concentration des solutions 
de caséine était déterminée, après filtration sur filtres $0,45 \mu \mathrm{m}$ (Millipore Corp., Bedford, Ma.), en utilisant le coefficient d'extinction $\underset{\mathrm{E}}{1 \mathrm{mg} / \mathrm{ml}} \underset{280 \mathrm{~nm}}{28,46}$ (SWAISGOOD, 1982).

Action de la phosphatase acide de pomme de terre (EC. 3.1.3.2.)

La phosphatase utilisée était commercialisée par Sigma (St. Louis, Mo.). L'enzyme était en suspension à $1 \mathrm{mg} / \mathrm{ml}$ dans $\left(\mathrm{NH}_{4}\right)_{2} \mathrm{SO}_{2} 3,2 \mathrm{M}, 1 \%$ sérum albumine, à $\mathrm{pH} 6,0$.

Initialement, la protéine était dissoute dans un tampon acétate de sodium $50 \mathrm{mM}, \mathrm{pH} 5,2$, selon la technique d'Alvarez (1962). Ces conditions ont été modifiées comme décrit dans le texte.

Action de la trypsine (EC. 3.4.21.4.)

L'enzyme (traité par le TPCK) provenait de chez Worthington (Freehold, NJ). La réaction était conduite à température ambiante pendant $14 \mathrm{~h}$ en tampon phosphate $100 \mathrm{mM}, 0,1 \% \mathrm{NaN3}, \mathrm{pH} 7,5$, caséine $\beta$ : $10 \mathrm{mg} / \mathrm{ml}$.

\section{Dosages d'acides aminés}

Ils étaient effectués sur un analyseúr LC 5000 (Biotronik, Munich, R.F.A.) équipé d'une colonne $3,2 \times 200 \mathrm{~mm}$. La composition en acides aminés des peptides était suffisante pour leur assigner une localisation dans la séquence de la caséine $\beta$ (RibadeAu Dumas et al., 1972).

Electrophorèse en gel d'acrylamide-agarose pH 8,6

Les gels ( $2 \mathrm{~mm}$ d'épaisseur) ont été préparés selon la technique d'URIEL (1966) modifiée par GRIPON et al. (1975). Les échantillons étaient préparés à une concentration finale variant entre $0,1 \%$ et $1 \%$ dans une solution d'urée 4,5 M, $2 \%$ agarose et $5 \%$ 2-mercaptoéthanol. $20 \mu 1$ étaient déposés.

La migration se faisait à $15^{\circ} \mathrm{C}$ sous une tension de $25 \mathrm{~V} / \mathrm{cm}$ pendant environ $2 \mathrm{~h}$. La coloration des gels, après fixation au TCA $12 \%$, était assurée par du bleu de Coomassie R 250.

\section{Dosage du phosphore}

Le phosphore a été dosé selon la méthode décrite par BAmann et al. (1948).

\section{$R P-H P L C$}

Les séparations étaient effectuées sur une colonne $\mu$-Bondapak $\mathrm{C} 18$ (Waters, Milford, Ma.). L'équipement consistait en un système contrôleur 720 , deux pompes $6000 \mathrm{~A}$, un injecteur U6K (Waters) et un spectrophotomètre à longueur d'onde variable CE 2023 (Cecil, Cambridge, Angleterre). La colonne était équilibrée avec le solvant A (phosphate de potassium $10 \mathrm{mM}, \mathrm{pH} 6,5$ ) et l'élution était obtenue par un gradient linéaire de $60 \mathrm{~min}$, de $100 \%$ de solvant $\mathrm{A}$ à $100 \%$ de solvant $\mathrm{B}\left(60 \% \mathrm{CH}_{3} \mathrm{CN}\right.$ et $40 \% \mathrm{~A}$, ajusté à $\left.\mathrm{pH} 6,5\right)$. La colonne et les solvants étaient immergés dans un bain à $40^{\circ} \mathrm{C}$. Le débit était de $1 \mathrm{ml} / \mathrm{min}$. L'absorption des peptides était enregistrée à $220 \mathrm{~nm}$. 


\section{Résultats}

\section{A. Mise au point des conditions de déphosphorylation}

A la suite des travaux de BRIGNON et al. (1977) sur la caséine $\alpha_{\$ 2}$, nous avons utilisé pour déphosphoryler la caséine $\beta$ bovine la phosphatase acide de pomme de terre comme décrit dans le paragraphe « Matériel et méthodes». L'inhibition de la réaction était assurée par remontée du $\mathrm{pH}$ du milieu vers 10 .

Pour un rapport $\mathrm{E} / \mathrm{S}=1 / 900$ et à $\mathrm{pH} 5,2$, la solution de caséine $\beta$ se troublait au bout de 5 à $10 \mathrm{~min}$. Au cours de la réaction, le trouble s'intensifiait et, au bout d'environ $60 \mathrm{~min}$, un précipité se formait. La réaction était suivie par électrophorèse en acrylamide-agarose pH 8,6 (fig. 1a). On constatait que la bande correspondant à la caséine $\beta$ disparaissait rapidement et que, parallèlement, des bandes de mobilités électrophorêtiques moindres apparaissaient. Cette évolution était stoppée à partir du temps correspondant à la précipitation du substrat.

La même expérience a été réalisée dans un boyau de dialyse $(5 \mathrm{ml}$ de milieu réactionnel), contre 21 de tampon acétate de sodium $50 \mathrm{mM}$ pH 5,2. Le résultat fut strictement comparable. Ainsi, il semble que l'arrêt de la déphosphorylation soit dû à la précipitation du substrat (et non pas, par exemple, à une action inhibitrice du phosphate inorganique libéré). Par ailleurs, on peut penser que cette précipitation est bien due à une augmentation du $\mathrm{pH}_{4}$ par suite d'une déphosphorylation partielle et non à une baisse du $\mathrm{pH}$ du milieu réactionnel par exemple.

Afin d'avoir une réaction plus complète, le $\mathrm{pH}$ du milieu a été remonté à 5,8 après $30 \mathrm{~min}$ de réaction. Dans ces conditions, le trouble disparaissait définitivement et, comme on le constate sur la figure $1 \mathrm{~b}$, la réaction se poursuivait jusqu'à l'accumulation d'une bande très majoritaire. L'électrophorèse de la figure $1 \mathrm{~b}$ appelle cependant une remarque : 5 bandes sont clairement mises en évidence au cours de la réaction, au lieu des 6 attendues (qui correspondraient à des protéines ayant de 5 à 0 phosphosérines). Trois hypothèses étaient envisageables :

- la caséine $\beta$ utilisée ne contenait que 4 phosphosérines; minés ;

- 4 seulement des 5 groupements phosphates auraient été totalement éli-

- la bande correspondant à 4 phosphosérines par molécule était confondue avec celle correspondant à la caséine $\beta$ intacte.

Pour tenter de lever l'ambiguïté, nous avons refait une électrophorèse en diminuant le rapport E/S et en déposant des quantités moindres sur le gel. Ainsi qu'on peut le voir sur la figure 2,6 bandes sont visibles dans ces conditions. Ainsi, seule la troisième hypothèse était recevable. On peut suggérer que le groupement phosphate éliminé le plus rapidement (et entraînant l'apparition de la bande $4 \mathrm{P}$ ) est celui qui est lié à la sérine 35 (qui est isolée). La proximité des 4 autres résidus phosphorylés (positions $15,17,18$ et 19) rend vraisemblablement leur déphosphorylation plus difficile.

Nous avons confirmé par dosage du phosphore que la bande la moins mobile en électrophorèse à $\mathrm{pH} 8,6$ correspondait effectivement à la caséine $\beta$ entièrement déphosphorylée. Ce dosage a été pratiqué sur l'échantillon 19 de la figure $1 \mathrm{~b}$ après dialyse contre de l'eau distillée à $4^{\circ} \mathrm{C}(5 \mathrm{ml}$ contre $6 \times 21$ d'eau $)$ durant $24 \mathrm{~h}$. La protéine était précipitée et a été resolubilisée par adjonction d'urée 


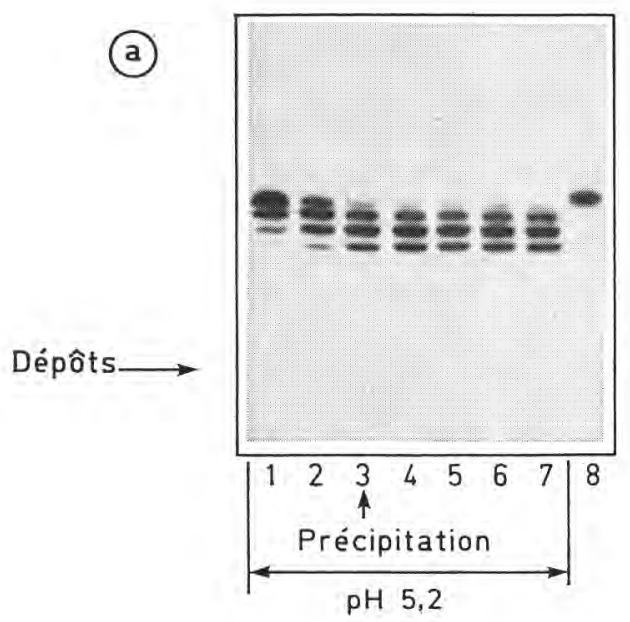

Fig. 1

Influence du pH et de la précipitation du substrat sur la déphosphorylation de la caséine $\beta$ par la phosphatase de pormme

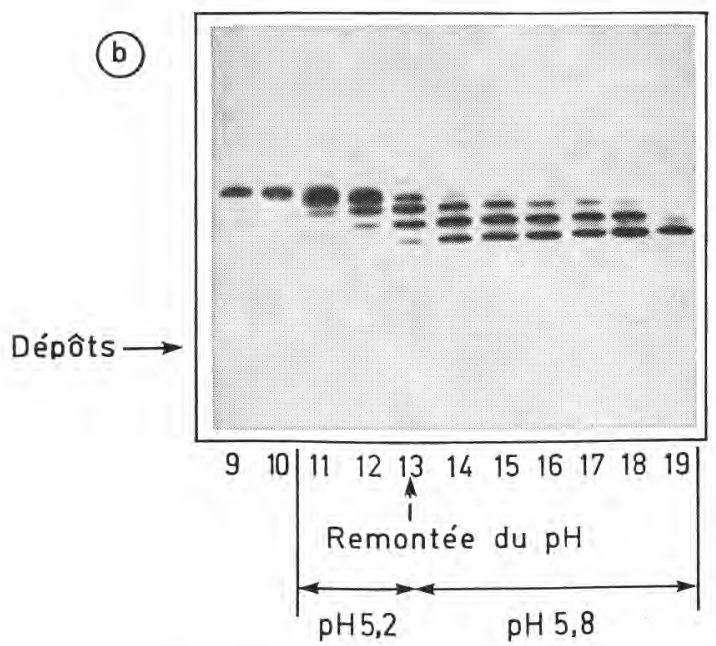
de terre $\left(E . S .=1 / 900 ; 30^{\circ} \mathrm{C}\right.$; acétate de sodium $50 \mathrm{mM}$ ).

a: Précipitation du substrat entre les prélèvements $20 \mathrm{~min}$ et $60 \mathrm{~min}$ (Dépôts 2 et 3 ) : $1: 10$ min de réaction ; 2 : $20 \mathrm{~min} ; 3: 1 \mathrm{~h} ; 4: 2 \mathrm{~h}$; $5: 4 h ; 6: 8 h ; 7: 24 h$; 8 : témoin caséine $\beta$-native pH 10,0 .

$b$ : Déphosphorylation sans précipitation du substrat : 9 : témoin caséine $\beta$-native, $p H$ 5,$2 ; 10$ : témoin caséine $\beta$ native, $\mathrm{pH} 10,0 ; 11$ : $5 \mathrm{~min}$ de réaction ; $12: 10 \mathrm{~min}$; $13: 20 \mathrm{~min} ; 14: 1 \mathrm{~h} ; 15:$ $2 h ; 16: 4 h ; 17: 8 h$; $18: 12 \mathrm{~h} ; 19: 24 \mathrm{~h}$.

Influence of $\mathrm{pH}$ and precipitation of the substrate on the action of potato acid phosphatase on $\beta$-casein $\left(E / S=1 / 1900 ; 50 \mathrm{mM}\right.$ sodium acetate $\left.; 30^{\circ} \mathrm{C}\right)$.

$a$ : Dephosphorylation with precipitation of the substrate between $20 \mathrm{~min}$ and $60 \mathrm{~min}$ of reaction (samples 2 and 3 ) : 1: reaction time $10 \mathrm{~min} ; 2: 20 \mathrm{~min} ; 3: 1 \mathrm{~h} ; 4: 2 \mathrm{~h}$; $5: 4 h ; 6: 8 h ; 7: 24 h ; 8:$ native $\beta$-casein, $p H 10.0$.

$b$ : Dephosphorylation without precipitation of the substrate: 9 : native $\beta$-casein, $p H 5.2$; 10: native $\beta$-casein, $p H 10.0 ; 11$ : reaction time $5 \mathrm{~min} ; 12: 10 \mathrm{~min} ; 13: 20 \mathrm{~min} ; 14$ : $1 \mathrm{~h} ; 15: 2 \mathrm{~h} ; 16: 4 \mathrm{~h} ; 17: 8 \mathrm{~h} ; 18: 12 \mathrm{~h} ; 19: 24 \mathrm{~h}$. 


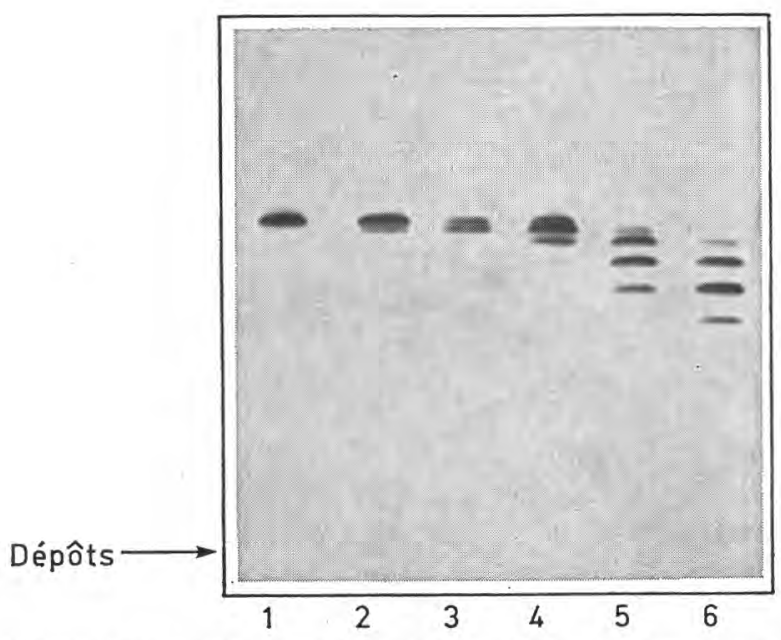

Fig. 2

Temps initiaux de la cinétique de déphosphorylation delacaséine $\beta$ par la phosphatase acide de pomme de terre $(E / S=1 / 2000$; $30^{\circ} \mathrm{C}$; acétate de sodium $50 \mathrm{mM}$, pH 5,8).

1: témoin caséine $\beta$ native, $p H$ 10,$0 ; 2: 2$ min de réaction; 3 : $5 \mathrm{~min} ; 4: 10 \mathrm{~min}$; 5: $20 \mathrm{~min}$; $6: 45 \mathrm{~min}$.

Initial times of desphosphorylation of $\beta$-casein by potato acid phosphatase $(E / S=1 / 2000$; $50 \mathrm{mM}$ sodium acetate, $\mathrm{pH} 5.8 ; 30^{\circ} \mathrm{C}$ ).

1: native $\beta$-casein at $p H 10.0 ; 2$ : reaction time $2 \mathrm{~min} ; 3: 5 \mathrm{~min} ; 4: 10 \mathrm{~min} ; 5: 20 \mathrm{~min}$; 6: 45 min.

TABLEAU I

Résultats obtenus pour le dosage du phosphore dans l'échantillon 19 (fig. 1b) (1) $0,5 \mathrm{ml}$ de l'échantillon 19 après $24 \mathrm{~h}$ de dialyse à $4^{\circ} \mathrm{C}$ contre de l'eau $+0,5 \mathrm{ml}$ d'eau ; (2) $1 \mathrm{ml}$ de l'échantillon 19 après $24 \mathrm{~h}$ de dialyse à $4^{\circ} \mathrm{C}$ contre de l'eau ; (3) $1 \mathrm{ml}$ tampon acétate de sodium après $24 \mathrm{~h}$ de dialyse à $4^{\circ} \mathrm{C}$ contre de l'eau; (4) caséine $\beta$ intacte après $24 \mathrm{~h}$ de dialyse à $4^{\circ} \mathrm{C}$ contre de l'eau

Determination of phosphorus in sample 19 (fig. 1b)

(1) 0,5 ml of sample 19 after 24 dialysis at $4^{\circ} \mathrm{C}$ against water + 0,5 ml water ; (2) $1 \mathrm{ml}$ of sample 19 after $24 \mathrm{~h}$ dialysis at $4^{\circ} \mathrm{C}$ against water ; (3) $1 \mathrm{ml}$ acetate buffer after $24 \mathrm{~h}$ dialysis at $4^{\circ} \mathrm{C}$ against water; (4) $\beta$-casein after $24 \mathrm{~h}$ dialysis at $4^{\circ} \mathrm{C}$ against water

\begin{tabular}{|c|c|c|c|c|}
\hline & \multicolumn{2}{|c|}{$\mathrm{DO}_{650}$} & \multicolumn{2}{|c|}{$\begin{array}{l}\text { Atomes de phosphore/mole } \\
\text { de protéine }\end{array}$} \\
\hline & essai 1 & essai 2 & essai 1 & essai 2 \\
\hline 1 & 0,020 & 0,017 & 0,1 & 0,1 \\
\hline 2 & 0,025 & 0,024 & 0,1 & 0,1 \\
\hline 3 & 0,016 & 0,020 & - & - \\
\hline 4 & 0,427 & 0,433 & 4,6 & 4,6 \\
\hline
\end{tabular}


(concentration finale : $4 \mathrm{M}$ ). Le tableau 1 récapitule les résultats obtenus. Pour la caséine $\beta$ intacte, le nombre d'atomes de phosphore trouvé par mole de caséine est de 4,6, ce qui est relativement satisfaisant. Pour l'échantillon 19, il est de 0,1 . L'analyse densitométrique du gel de la figure $1 \mathrm{~b}$ indique que les deux bandes visibles pour cet échantillon sont dans un rapport $9 / 1$, ce qui est parfaitement cohérent avec le dosage précédemment réalisé $(0,1=0,9 \times 0+0,1 \times 1)$. Ainsi, nons pouvons conclure que la bande majoritaire la moins mobile correspond à de la caséine $\beta$ entièrement déphosphorylée et que la bande contaminante correspond à de la caséine $\beta$ à laquelle il ne reste qu'une phosphosérine.

Sur la figure $1 \mathrm{~b}$, on peut observer, pour les temps longs de réaction (échantillons 18 et 19, par exemple), une protéolyse parallèle à la déphosphorylation.

Il apparaît que la précipitation de la caséine $\beta$ stoppe la protéolyse (comparaison échantillons 7 -fig. 1a et 19-fig. 1b). Nous avons essayé d'inhiber cette protéolyse en testant pour deux temps longs de déphosphorylation (24 et $48 \mathrm{~h}$ ), et avec un rapport E/S supérieur, cela afin de mieux visualiser cette protéolyse parallèle, l'effet de 2 inhibiteurs à spectre large : l'EDTA et le PMSF (concen-

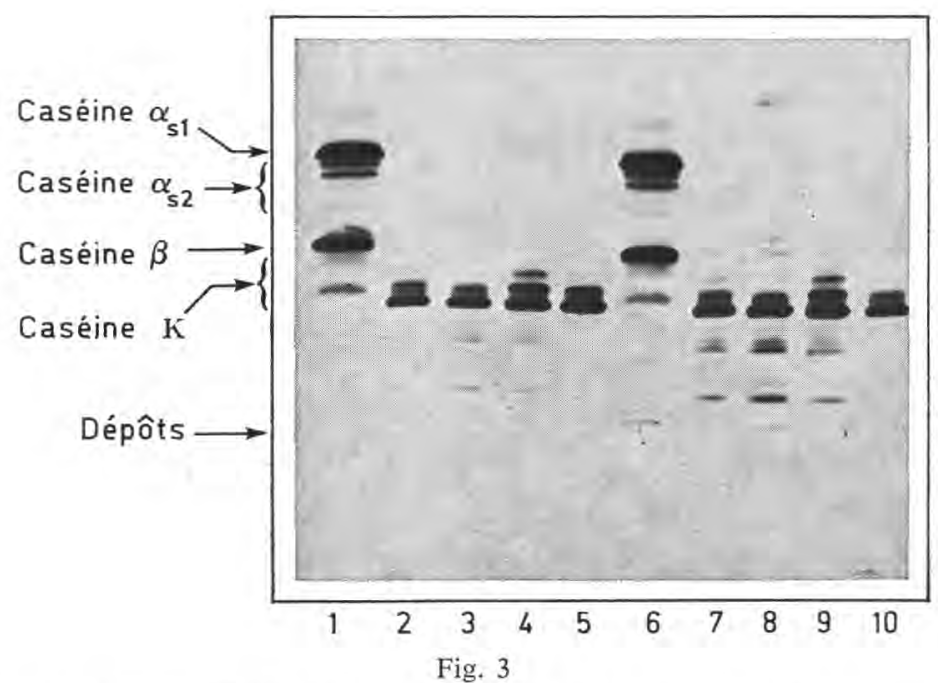

Inhibition de la protéolyse parallèle à la déphosphorylation de la caséine $\beta$ par la phosphatase acide de pomme de terre $\left(30^{\circ} \mathrm{C}\right.$; acétate de sodium $\left.50 \mathrm{mM} ; \mathrm{pH} 5,8\right)$.

1 et $6:$ caséine entière; $2:$ temps de réaction $24 h, E / S=1 / 2000 ; 3:$ temps de réaction $24 \mathrm{~h}, \mathrm{E} / \mathrm{S}=1 / 500,1 \mathrm{mM}$ EDTA final $; 4:$ temps de réaction $24 \mathrm{~h}, \mathrm{E} / \mathrm{S}=1 / 500 ; 5:$ temps de réaction $24 \mathrm{~h}, \mathrm{E} / \mathrm{S}=1 / 500,1 \mathrm{mM}$ PMSF final; $7:$ temps de réaction $48 \mathrm{~h}, \mathrm{E} / \mathrm{S}=1 / 2000$; 8: temps de réaction $48 \mathrm{~h}, E / S=1 / 500 ; 9$ : temps de reaction $48 \mathrm{~h}, E / S=1 / 500$; 10 : temps de réaction $48 \mathrm{~h}, E / S=1 / 500,1 \mathrm{mM} P M S F$ final.

Inhibition of parallel proteolysis during the dephosphorylation of $\beta$-casein by potato acid phosphatase $\left(50 \mathrm{mM}\right.$ sodium acetate; $\left.\mathrm{pH} 5.8 ; 30^{\circ} \mathrm{C}\right)$.

1 and $6:$ whole casein $; 2:$ reaction time $24 \mathrm{~h}, \mathrm{E} / \mathrm{S}=1 / 2000 ; 3:$ reaction time $24 \mathrm{~h}, \mathrm{E} / \mathrm{S}=$ $1 / 500,1 \mathrm{mM}$ EDTA (final concentration) ; $4:$ reaction time $24 \mathrm{~h}, \mathrm{E} / \mathrm{S}=1 / 500 ; 5:$ reaction time $24 \mathrm{~h}, E / S=1 / 500,1 \mathrm{mM} P M S F$ (final concentration) $; 7:$ reaction time $48 \mathrm{~h}, E / S=$ $1 / 2000 ; 8:$ reaction time: $48 \mathrm{~h}, \mathrm{E} / \mathrm{S}=1 / 500,1 \mathrm{mM}$ EDTA (final concentration) ; 9 : reaction time $48 \mathrm{~h}, E / S=1 / 500,1 \mathrm{mM} P M S F$ (final concentration). 

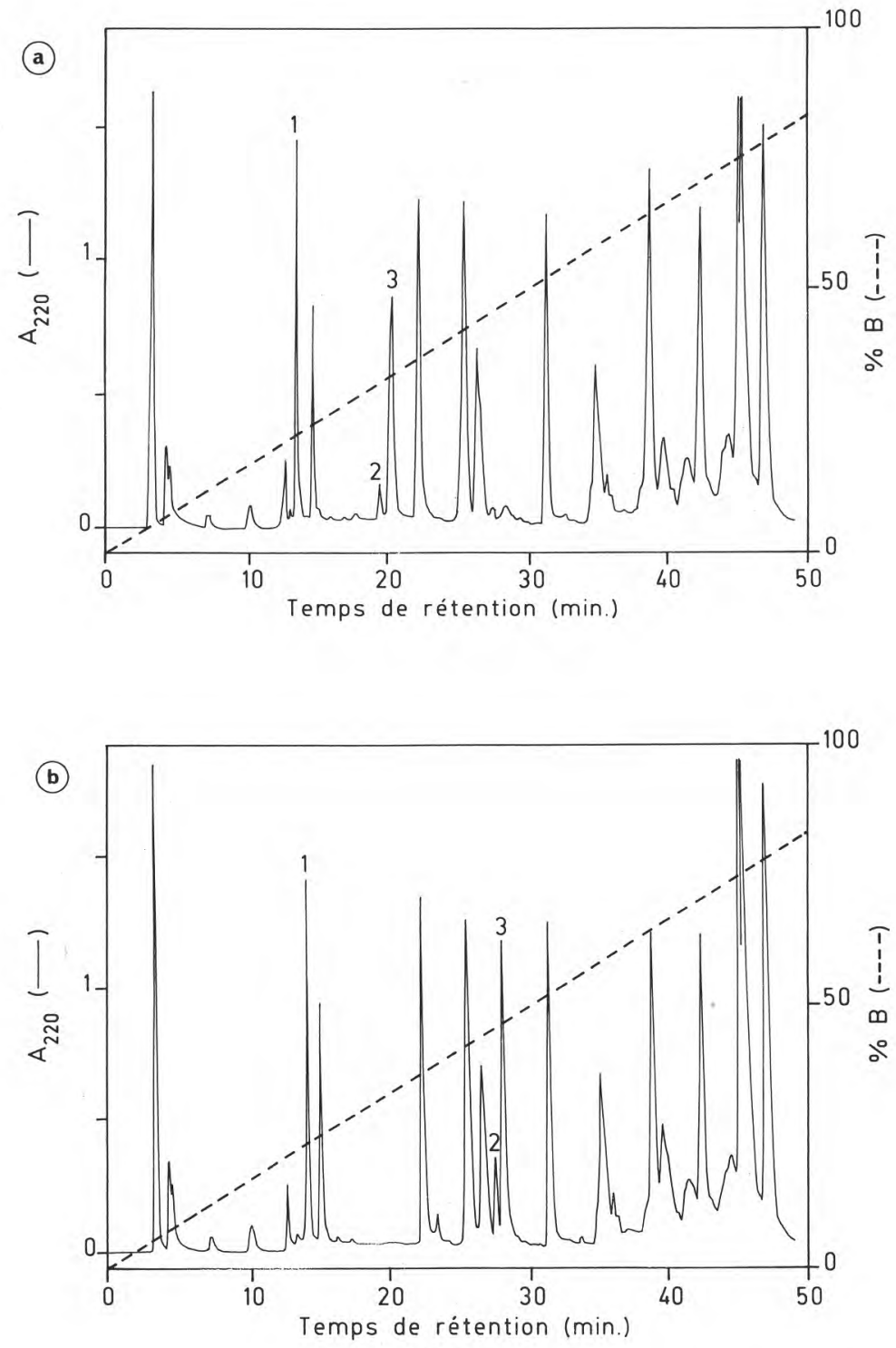

Fig. 4

Comparatson des hydrolysats trypsiques de caséine $\beta$ native (a) et totalement déphosphorylée $(b)$ en RP-HPLC. Débit : $1 \mathrm{ml} / \mathrm{min}$. Colonne : $\mu$ Bondapak C18.

Solvant $A$ : phosphate de $K 10 \mathrm{mM} \mathrm{pH} 6,5$; solvant $B: 60 \% \mathrm{CH}_{3} \mathrm{CN}+40 \%$ solvant $A$. Pic 1: peptide $33-48$; pic 2 : peptide $2-25$; pic 3 : peptide $1-25$.

Comparison of tryptic peptide mappings of native $(a)$ and dephosphorylated $(b) \beta$-casein by $R P-H P L C$.

Column : $\mu$ Bondapak C18; flow-rate: $1 \mathrm{ml} / \mathrm{min}$; solvent $A: 10 \mathrm{mM} \mathrm{K}$ phosphate, $\mathrm{pH} 6.5$; solvent $B: 60 \% \mathrm{CH}_{3} \mathrm{CN}+40 \%$ solvent $A ; 1$ : peptide $33-48 ; 2$ : peptide $2-25 ; 3$ : peptide $1-25$. 
tration finale dans les 2 cas : $1 \mathrm{mM}$ ). Sur la figure 3 , on voit clairement que le PMSF inhibe totalement les réactions de protéolyse parallèles. Au contraire, l'EDTA semble les favoriser légèrement.

$\mathrm{Au}$ vu de ces résultats, les conditions expérimentales que nous avons adoptées pour la déphosphorylation totale de la caséine $\beta$ sont les suivantes : $\mathrm{E} / \mathrm{S}=$ $1 / 2000$; caséine $\beta$ solubilisée dans du tampon citrate de sodium $50 \mathrm{mM}$ pH 5,8 $+1 \mathrm{mM}$ PMSF ; $24 \mathrm{~h}$ d'action à $30^{\circ} \mathrm{C}$. C'est sur un échantillon déphosphorylé dans ces conditions qu'ont été poursuivies les études en HPLC.

\section{B. Comparaison des hydrolysats trypsiques de caséine $\beta$ intacte et totalement déphosphorylée}

Il s'agissait de voir si la comparaison des «cartes peptidiques.» (réaliseés dans les mêmes conditions) d'une protéine intacte et de la même protéine après déphosphorylation permettait de mettre en évidence directement les peptides correspondant aux zones phosphorylées de la protéine. Une telle démarche a été appliquée, par exemple, aux peptides contenant de la cystéine par KoEPPE et al. (1985).

L'enzyme que nous avons utilisée était la trypsine. Les peptides séparés par RP-HPLC ont été identifiés sans ambiguité par leur composition en acides aminés. La comparaison des chromatogrammes (a) et (b) de la figure 4 montre que tous les peptides non phosphorylés ont des temps de rétention identiques. Si l'on s'intéresse aux phosphopeptides, 3 molécules sont à considérer : les peptides 1-25 et 2-25 (pics 2 et 3 respectivement), ne différant que par une Arg $\mathrm{N}$-terminale et possédant chacune 4 phosphosérines, et le peptide 33-48 (pic 1) qui n'en contient qu'une. On observe pour les peptides $2-25$ et 1-25 que la déphosphorylation s'accompagne d'une augmentation très nette du temps de rétention $(\Delta \mathrm{tr}=$ $7,6 \mathrm{~min})$.

L'augmentation est beaucoup plus faible $(0,4 \mathrm{~min}$ environ) pour le peptide 33-48. Sur trois passages, les variations observées pour les pics $[1,2,3]$ entre la protéine intacte et la protéine déphosphorylée étaient absolument comparables.

\section{Discussion}

Il apparaît clairement, du fait de la grande reproductibilité de la RP-HPLC, qu'une différence systématique, même faible, du temps de rétention d'un peptide (entre 2 hydrolysats réalisés avant et après déphosphorylation) permet de conclure quant à la présence initiale de groupements phosphates. C'est donc là un moyen simple et rapide d'identifier dans un mélange complexe les molécules porteuses de telles modifications post-traductionnelles.

Compte tenu de la nature des paramètres influençant la séparation de solutés sur les phases inverses, il n'est pas surprenant que la disparition de groupements très polaires augmente la rétention d'un composé. Si l'on se réfère à l'approche de MeEK (1980) dans laquelle la rétention d'un peptide peut s'exprimer comme la somme arithmétique des contributions de chaque résidu, des groupements ter- 
minaux et des substitutions latérales, approche qui implique donc que les interactions intramoléculaires ne jouent aucun rôle, on peut conclure que, dans nos conditions d'élution, chaque groupement phosphate substituant une sérine pour les peptides $1-25$ et $2-25$ a une contribution de $1,9 \mathrm{~min}$ (ou $1,9 \mathrm{ml}$ si l'on parle en terme de volume d'élution). Cette contribution n'est par contre que de $0,4 \mathrm{~min}$ pour le peptide 33-48. Ainsi, une même substitution latérale a, dans des conditions identiques, une influence différente sur 2 molécules. Ceci va à l'encontre de l'approche de MeEK, et, bien que cette dernière soit intéressante, elle s'avère difficilement utilisable dans un cadre très général. Ce point était d'ailleurs déjà apparu à cet auteur puisque, par rapport à l'article de base (MeEK, 1980), de nouveaux coefficients de contribution avaient été recalculés à partir d'un nombre supérieur de données (MEek et RossetTi, 1981) afin d'en généraliser l'utilisation. Il a de toute façon été clairement établi que ces coefficients ne sont pas transposables à des conditions d'élution différant, même faiblement, de celles dans lesquelles ils ont été déterminés (BROWNE et al., 1982).

Nous pouvons donc conclure que, s'il est aisé de déterminer les peptides phosphorylés, il est par contre impossible de prédire le nombre exact de résidus phosphorylés portés par les molécules ainsi mises en évidence.

Reçu le 9-7-1985.

Accepté pour publication le 21-11-1985.

\section{Références bibliographiques}

Alvarez E., 1962. The kinetics and mechanism of the hydrolysis of phosphoric acid ester by potato acid phosphatase. Biochim. Biophys. Acta, 59, 663-672.

Bamann E., Novotny E., Rohr L., 1948. On the colorimetric determination of phosphoric acid. Chem. Bericht., 81, 438-441.

Brignon G., Ribadeau Dumas B., Mercier M.J., Pélissier J.P., 1977. Complete amino acid sequence of bovine as2-casein. FEBS Lett., 76, 274-279.

Browne C., BennetT H., Solomon S., 1982. The isolation of peptides by high-performance liquid chromatography using predicted elution positions. Anal. Biochem., 124, 201-208.

Gripon J.C., Desmazeaud M.J., Le Bars D., Bergère J.L., 1975. Etude du rôle des microorganismes et des enzymes au cours de la maturation des fromages. II. Influence de la présure commerciale. Lait, 55 (548), 502-516,

KoEpre R., Haw J., PAczkowski J., 1985. On the recovery of Cys-containing peptides during peptide mapping by HPLC : tryptic peptides of trp-tRNA synthetase of $E$. coli. FEBS Lett., 183 (2), 313-316.

MEEK J., 1980. Prediction of peptide retention times in high pressure liquid chromatography on the basis of amino acid composition. Proc. Natl. Acad. Sci., USA, 77 (3), 1632-1636.

MEex J., RossetTi Z., 1981. Factors affecting retention and resolution of peptides in high performance liquid chromatography. J. Chromatogr., 211, 15-28.

Mercier J.C., Maubots J.L., Poznanski S., Ribadeau Dumas B., 1968. Fractionnement préparatif des caséines de vache et de brebis par chromatographie sur DEAE-cellulose en milieu urée et 2-mercaptoéthanol. Bull. Soc. Chim. Biol., 50, 521-530.

Ribadeau Dumas B., Brignon G., Grosclaude F., Mercier J.C., 1972. Structure primaire de la caséine $\beta$-bovine. Eur. J. Biochem., 25, 505-514.

Swaisgood H., 1982. Chemistry of milk proteins. In : Developments in Dairy Chemistry, édité par Fox P.F., Applied Science Publishers, N.Y., 1-59.

URIEL J., 1966. Méthode d'électrophorèse dans des gels d'acrylamide-agarose. Bull. Soc. Chim. Biol., 48, 969-982. 